\title{
CARATERIZAÇÃO DAS ATIVIDADES LÚDICO- MOTORAS EM CRIANÇAS DA CIDADE DA GUARDA. ASSOCIAÇÃO ENTRE PRÁTICA LÚDICA, IMC E APTIDÃO FÍSICA
}

CHARACTERIZATION OF MOTOR PLAY ACTIVITIES IN CHILDREN FROM THE CITY OF GUARDA. ASSOCIATION BETWEEN PLAYFUL PRACTICE, BMI AND PHYSICAL FITNESS

CARACTERIZACIÓN DE LAS ACTIVIDADES LÚDICAS Y MOTORAS EN NIÑOS DE LA CIUDAD DE GUARDA. ASOCIACIÓN ENTRE PRÁCTICA LÚDICA, IMC Y APTITUD FÍSICA

\author{
Nuno Serra (nserra@ipg.pt)* \\ Andreia Silva (4andreiasilva@gmail.com)** \\ Carlos Marta (carlosmarta@ipg.pt) ${ }^{\star \star *}$ \\ Carolina Vila-Chã (cvilacha@ipg.pt)****
}

\section{RESUMO}

Assiste-se, em Portugal, à preocupação com o crescente sedentarismo entre as crianças em idade escolar associado à elevada taxa de obesidade infantil e ao aumento de iliteracia motora. Paradoxalmente, parecem colocar-se cada vez mais obstáculos às oportunidades de as crianças realizarem atividades lúdicas. O estudo tem como objetivo verificar as associações entre o número e tipo de jogos realizados por sujeitos pré-púberes, as componentes de aptidão física (ApF) e o índice de massa corporal (IMC). A amostra foi constituída por 108 crianças, de 10 e 11 anos, de um agrupamento de escolas da área urbana da Guarda. Para a recolha de dados referentes aos jogos praticados foi utilizada uma entrevista padronizada, adaptada de Serra (1992). A avaliação da ApF incluiu testes de capacidade aeróbia, flexibilidade, força e resistência muscular, através dos protocolos da bateria de testes Fitnessgram e velocidade (20m). Os dados revelaram o quase desaparecimento de jogos de lançamento no tempo livre das crianças, registando-se também valores muito baixos nos de saltos e outras práticas lúdicas com bola (exceptuando-se os de cariz desportivo). As crianças desta amostra com massa corporal e IMC mais elevados praticam menos jogos de atividade física, nomeadamente no 
que concerne aos jogos com bola (desportivos e tradicionais) e de corrida e perseguição. Os dados realçaram a associação entre o tipo e quantidade de jogos realizados em cada um dos contextos analisados e algumas componentes da ApF.

Palavras-chave: Jogos, Aptidão Física, Índice de massa Corporal

\section{ABSTRACT}

Recently in Portugal, there is concern about the growing sedentary behaviour among school-aged children, which has been associated with obesity and motor illiteracy in youth people. Paradoxically, there seem to be more and more obstacles to the opportunities for children to engage in playful activities. The aim of the study was to verify the associations between the number and type of games performed by prepubescent subjects with physical fitness components (ApF) and body mass index (BMI). The sample consisted of 108 children, aged 10 and 11, from schools in the urban area of Guarda. A standardized interview, adapted from Serra (1992), was used to gather data on the games practiced. ApF's assessment included tests of aerobic capacity, flexibility, strength, and muscular endurance, through Fitnessgram and velocity $(20 \mathrm{~m})$ battery protocols. The data revealed the withdrawal of throwing games in the children's free time, with very low values of jumps and other playful practices (except sports ones). In this sample, the children with higher body mass and BMl practiced less physical activity games, especially in relation to ball games (sport and traditional) and running and chasing. The data highlighted the association between the type and quantity of games performed in each of the contexts analyzed and some components of the ApF.

keywords: Games, physical fitness, body mass index

\section{RESUMEN}

Se asiste, en Portugal, a la preocupación por el creciente sedentarismo entre los niños en edad escolar asociado a la elevada tasa de obesidad infantil y al aumento de analfabetismo motor. Paradójicamente, parecen 
colocarse cada vez más obstáculos a las oportunidades de los niños para realizar actividades lúdicas. El estudio tiene como objetivo verificar las asociaciones entre el número y tipo de juegos realizados por sujetos prepúberes, los componentes de aptitud física (APF) y el índice de masa corporal. La muestra fue constituida por 108 niños, de 10 y 11 años, de una agrupación de escuelas del área urbana de Guarda. Para la recogida de datos referentes a los juegos practicados se utilizó una entrevista estandarizada, adaptada de Serra (1992). La evaluación de la APF incluyó pruebas de capacidad aeróbica, flexibilidad, fuerza y resistencia muscular, a través de los protocolos de la batería de pruebas Fitnessgram y velocidad (20m). Los datos revelaron el casi desaparecimiento de los juegos de lanzamiento en el tiempo libre de los niños, registrándose también valores muy bajos en los de saltos y otras prácticas lúdicas con balón (exceptuando los de cariz deportivo). Los niños de esta muestra con masa corporal e IMC más elevados practican menos juegos de actividad física, especialmente en lo que concierne a los juegos con pelota (deportivos y tradicionales) y de carrera y persecución. Los datos realzaron la asociación entre el tipo y cantidad de juegos realizados en cada uno de los contextos analizados y algunos componentes de la APF.

Palabras clave: Juegos, aptitud física, índice de masa corporal

\footnotetext{
* Professor Adjunto da Escola Superior de Educação, Comunicação e Desporto do Instituto Politécnico da Guarda, Portugal. Membro da Unidade de Investigação para o Desenvolvimento do Interior (UDI). Doutorado em Ciências da Atividade Física e do Desporto pela Universidade de León.

** Aluna da Licenciatura em Desporto do Instituto Politécnico da Guarda

*** Doutorado em Ciências do Desporto pela Universidade da Beira Interior. Membro da UDI.

**** Professora Adjunta da Escola Superior de Educação, Comunicação e Desporto do Instituto Politécnico da Guarda, Portugal. Membro efetivo da Research Centre in Sports Sciences, Health Sciences and Human Development, CIDESD, Portugal. Membro da UDI. Doutorada em Engenharia Biomédica pela Universidade do Porto.
} 


\section{INTRODUÇÃO}

A importância do jogo no desenvolvimento global e harmonioso do ser humano foi reconhecida, em todas as épocas históricas, por psicólogos, pedagogos e antropólogos. O mesmo não se tem passado, porém, no caso específico dos jogos tradicionais (JT), estudados apenas, numa perspetiva antropológica. Efetivamente, nestas práticas lúdicas, portadoras de particularidades locais e regionais e transmitidas de geração em geração, ao longo de décadas e séculos, tal evidência não tem merecido a devida atenção dos investigadores. E muito menos têm suscitado o interesse dos estudiosos os benefícios do exercício de alguns JT no domínio específico das capacidades físicas e motoras.

Entendemos que a concretização de estudos neste âmbito se torna cada vez mais premente e relevante, num tempo em que os hábitos sedentários, a obesidade e as doenças correlacionadas se tornam realidades muito frequentes nas sociedades desenvolvidas (Carvalhal \& Silva, 2006).

É comummente reconhecido o papel que o desporto e as atividades físicas realizadas em ginásios e academias desempenham na prevenção desses comportamentos inapropriados, contribuindo para a melhoria da condição física das populações. Porém, em determinadas franjas etárias, mormente na infância e nas idades mais avançadas, verifica-se que o acesso às aludidas práticas lúdicas e desportivas nem sempre se torna fácil. O mesmo se passa em muitos meios rurais ou em populações citadinas social e economicamente desfavorecidas.

No caso do desporto, as exigências técnicas, físicas e motoras que a sua prática requer transformaram-no numa realidade algo seletiva. $\bigcirc$ caráter pouco inclusivo da solução desportiva afasta muitas crianças e jovens, que rapidamente derivam para uma dependência exagerada e doentia, porque isolada e inativa, das inovações tecnológicas.

Perante este quadro, vários autores sublinham a notória declinação da atividade física logo a partir da infância, quer em meninas, quer em meninos (Sallis, 2000; Telama \& Yang, 2000; Van Mechelen et. al., 2000). Em 1882, o padre Pedro Aloy, no seu livro "Recreios Collegiais...", descreve 150 práticas lúdicas, a maioria das quais com caraterísticas tradicionais, salientando que "...o recreio é o asylo da liberdade dos colegiais." Visando a livre ação dos alunos, a esmagadora maioria dos 
jogos mais adequados aos recreios "maiores" e "menores", em especial os de "inverno" e de "primavera", apresenta, na sua dinâmica e desenvolvimento, uma forte componente física e motora (p. V).

Todavia, do final do séc. XIX até aos nossos dias, aquela realidade mudou drasticamente. Com efeito, os jogos realizados pelos alunos nos intervalos das aulas são cada vez em menor número, com uma forte tendência para atividades que não exigem a intervenção da motricidade grossa. Registam-se hoje menos oportunidades para a realização de jogos e brincadeiras, práticas fundamentais para o desenvolvimento físico, social e mental das crianças (Bulut \& Yilmaz, 2008; Neto, 2008). Também outras atividades sedentárias, realizadas no computador ou na biblioteca, são agora oferecidas às crianças, em substituição das antigas práticas lúdico-desportivas de natureza física ou motora (Bragança et. al., 2008).

Beaulier e Ronné (2002:12) salientam que os jogos praticados pelas crianças constituem uma escola informal, "... onde o corpo e o espírito se educam numa progressão notável". É durante o tempo de recreio, nos logradouros das escolas, que os alunos estreitam as suas relações sociais e estabelecem, adotam ou modificam a regras dos seus jogos (Blatchford, 1998).

No domínio da atividade física, o recreio escolar tem sido referenciado por autoridades reguladoras da saúde como o tempo e o espaço mais profícuos à promoção da AF dos alunos (Pereira, 2001). Com efeito, além das aulas de Educação Física (inseridas no horário ou consideradas nas atividades de enriquecimento curricular) e do Desporto Escolar, que surge após o $1^{\circ}$ Ciclo do Ensino Básico, os recreios constituem uma oportunidade ímpar para a realização de práticas ativas (USDHHS, 2000; Ridgers et. al. 2005). Eles apresentam, em relação aos tempos de AF orientada, a grande vantagem de proporcionarem práticas escoIhidas pelas crianças, organizadas e desenvolvidas sem a intervenção dos adultos.

Do vasto leque de JT outrora conhecidos e praticados em todos os recreios, interessa destacar e adaptar, numa perspetiva eminentemente pedagógica e recreativa, os que apresentam uma forte componente de atividade física e motora diversificada. Referimo-nos a imensos jogos cujo desenvolvimento se estrutura em torno de ações de locomoção, corrida e perseguição, saltos, equilíbrio e demonstração de força corpo- 
ral.

Não temos conhecimento da existência de estudos que investiguem a associação entre a tipologia de prática lúdica, o IMC e a aptidão física numa amostra de pré-adolescentes na região da Guarda (Centro de Portugal). Assim, os objetivos deste estudo foram: i) verificar quais as atividades lúdico-motoras mais realizadas por alunos do $2^{\circ}$ ciclo do Ensino Básico, no recreio escolar e fora dele ii) perceber se existe uma associação entre o número e o tipo de jogos, a aptidão física e o IMC, em crianças pré-pubertárias da região da Guarda.

\section{METODOLOGIA}

\section{AMOSTRA}

A amostra foi constituída por 108 crianças (66 do género feminino e 42 do masculino), de 10 e 11 anos, com o valor médio de IMC de 19,17 \pm 6,16, de agrupamento de escolas da área urbana da Guarda, sem doenças pediátricas crónicas ou limitações ortopédicas. Os sujeitos integrantes da amostra e o professor de Educação física envolvido na recolha de dados foram previamente informados sobre a natureza e objetivos do estudo. Na sua concretização observaram-se os mais elevados índices de segurança e as recomendações éticas decorrentes da Declaração de Helsínquia.

\section{PRÁTICAS LÚDICO-MOTORAS}

A recolha de dados referentes aos jogos praticados foi realizada através de uma entrevista padronizada, adaptada de Serra (1992: 45-46), feita diretamente a cada sujeito pelo seu professor de educação física, no ano escolar de 2016/2017. Os entrevistados foram questionados acerca dos jogos que costumavam praticar no espaço escolar e noutros locais. As adaptações introduzidas à taxonomia do referido autor foram justificadas pela especificidade dos exercícios lúdicos que se pretendia investigar (somente os que provocavam um considerável desgas- 
te calórico). Por consequência, não foram consideradas no presente estudo as classes de jogos eletrónicos e de mesa, baseados apenas na motricidade fina. Cada uma das práticas lúdicas mencionadas pelos sujeitos da amostra foi agrupada numa das nove classes, mutuamente exclusivas, da tipologia adotada:

- Jogos de corrida e perseguição (CP), nos quais é utilizada a corrida para tocar ou agarrar outro(s);

- Danças e batimentos rítmicos (DA), movimentos ou batimentos ritmados, de mãos ou pés, acompanhados de uma fórmula rimada ou cantilena;

- Jogos desportivos com bola (DB), ou seja, formas de prática desportiva, mesmo que reduzidas ou rudimentares, com utilização de uma bola ou objeto volante;

- Jogos de descoberta (DE), consistindo em encontrar objeto(s) ou jogador(es) escondido(s);

- Jogos de dramatização (DR), situações de ficção, "faz de conta" ou representação de papéis;

- Jogos de lançamento em precisão (LA), práticas que se baseiam no lançamento de um ou mais objetos, procurando acertar num ou em diversos alvos;

- Jogos de locomoção (LC), cujo objetivo fundamental é procurar deslocar o próprio corpo ou o de outro(s), com e sem material apropriado;

- Outros jogos com bola (OB), isto é, jogos tradicionais em cuja prática se usa uma bola;

- Jogos de saltos (SA), exercícios cuja tarefa motora essencial é saltar ou saltitar.

\section{AVALIAÇÃO DA APTIDÃO FÍSICA}

Para a avaliação da aptidão física foram selecionados testes que incluíssem a avaliação da capacidade aeróbia, flexibilidade, força e resistência 
muscular, através dos protocolos da bateria FITNESSGRAM (Meredith \& Welk, 2007) e velocidade (20m). O coeficiente de correlação intraclasse (CCl) variou entre 0,93 e 0,99.

\section{MEDIÇÕES ANTROPOMÉTRICAS}

Todas as medições antropométricas foram realizadas de acordo com os padrões internacionais reconhecidos para o efeito (Marfell-Jones et. al., 2006). A massa corporal (kg) foi medida com aproximação de .10 kg, utilizando uma balança digital (Seca, modelo 841, Alemanha). Para avaliar a estatura (cm) foi usado um estadiómetro de precisão, com uma escala de intervalo de .10 cm (Seca, modelo 214, Alemanha).

\section{ANÁLISE ESTATÍSTICA}

Utilizaram-se métodos estatísticos padrão para as medidas de tendência central e de dispersão, tendo-se recorrido ao coeficiente de correlação Intraclasse $(\mathrm{CCl})$ para determinar o grau de confiabilidade nos testes selecionados. Devido à distribuição não normal de algumas variáveis (verificada através do teste de Kolmogorov-Smirnov) recorreuse ao coeficiente de correlação de Spearman, para obter o grau de associação entre as variáveis, tendo sido aferido o nível de significância estatística para $\mathrm{p} \leq .05$.

\section{RESULTADOS}

\section{PRÁTICAS LÚDICAS REFERENCIADAS POR CLASSE E CONTEXTO}

A tabela 1 elucida-nos que, em termos absolutos, o total de referências aos jogos e desportos realizados fora da escola (FE) atingiu um valor próximo do dobro dos praticados em meio escolar. Os jogos de corrida e perseguição (CP) surgem como os mais praticados nos dois contextos em análise, atingindo as taxas percentuais de 39,8\% no meio 
escolar (ME) e 20, 9\% fora da escola (FE).

Tabela 1 - Jogos referenciados por espaço

\begin{tabular}{|l|c|c|c|c|}
\hline \multirow{2}{*}{ Classes de jogos } & \multicolumn{4}{c|}{ Espaço } \\
\cline { 2 - 5 } & \multicolumn{2}{|c|}{ Escolar } & \multicolumn{2}{c|}{ Não Escolar } \\
\cline { 2 - 5 } & $n$ & $\%$ & $n$ & $\%$ \\
\hline Corrida e perseguição(CP) & 123 & 39,8 & 111 & 20,9 \\
\hline Danças e batimentos Rítmicos (DA) & 2 & 0,64 & 10 & 1,9 \\
\hline Jogos desportivos com bola (DB) & 63 & 20,4 & 85 & 15,9 \\
\hline Jogos de descoberta (DE) & 49 & 15,9 & 76 & 14,3 \\
\hline Jogos de dramatização (DR) & 10 & 3,2 & 32 & 6,1 \\
\hline $\begin{array}{l}\text { Jogos de lançamento em precisão } \\
\text { (LA) }\end{array}$ & 0 & 0 & 1 & 0,18 \\
\hline Jogos de locomoção (LC) & 38 & 12,3 & 105 & 19,7 \\
\hline Outros jogos com bola (OB) & 12 & 3,9 & 21 & 3,94 \\
\hline Jogos de saltos (SA) & 12 & 3,9 & 90 & 16,91 \\
\hline Total de referências & 309 & 100 & 531 & 100 \\
\hline
\end{tabular}

Os jogos desportivos com bola (DB) e os jogos de locomoção (LC) atingiram, também, valores elevados, quer no meio escolar $(20,4 \%$ e 12,3\%, respetivamente), quer fora desta instituição (15,9\% e 19,7\%, respetivamente). Deve salientar-se o facto de os jogos considerados nestas três classes se caraterizarem pelo seu caráter dinâmico e elevada exigência ao nível das capacidades condicionais e coordenativas.

Os dados revelaram valores percentuais baixos das categorias de jogos de dramatização (3,2\% em ME e 6,1\% FE) e outros com bola (3,9\% em qualquer dos meios em estudo). As danças e batimentos rítmicos (DA) mereceram referências com um valor percentual muito baixo (0,6\% em ME e 1,9 FE), registando-se o desaparecimento dos jogos de lançamento (LA) no meio escolar e apenas uma referência ao jogo do berlinde fora da escola $(0,2 \%)$.

Os jogos de saltos, cujas referências registaram um valor baixo na escola (3,9\%), atingiram uma percentagem bem mais elevada fora do contexto escolar (16,9\%). 
Associação entre jogos praticados e aptidão morfológica

Verificaram-se correlações significativas e negativas entre algumas práticas lúdicas realizadas em meio escolar e valores de aptidão morfológica. O IMC registou uma associação com os jogos desportivos com bola $(r=-0,258, p=0,007)$ e ainda com outros jogos com bola ( $r=$ $-0,196, p=0,042)$.

Analisando a associação entre a massa corporal e as práticas lúdicas de corrida e perseguição e o número total de jogos realizados em contexto escolar, verificou-se também uma correlação significativa e negativa ( $r=$ $-0,281, p=0,003$ e $r=-0,294, p=0,002$, respetivamente).

Tabela 2- Variáveis antropométricas e de aptidão física: Valor mínimo, máximo, média e desvio p
\begin{tabular}{|l|c|c|c|c|}
\hline Parâmetros & Mín & Máx & Média & DP \\
\hline Massa corporal & 24,0 & 65,0 & 38,533 & 9,5789 \\
\hline Estatura & 130 & 175 & 146,32 & 8,806 \\
\hline IMC & 15 & 30 & 19,17 & 6,155 \\
\hline Vaivém & 7 & 60 & 17,02 & 9,203 \\
\hline Flexibilidade Esquerda &, 0 & 30,0 & 17,956 & 6,4707 \\
\hline Flexibilidade Direita &, 0 & 30,0 & 18,599 & 6,3688 \\
\hline Extensão Braços & 0 & 55 & 10,72 & 10,707 \\
\hline Abdominais & 9 & 60 & 43,49 & 17,218 \\
\hline Velocidade & 3,54 & 5,24 & 4,2054 &, 37535 \\
\hline
\end{tabular}

\section{ASSOCIAÇÃO ENTRE JOGOS PRATICADOS E APTIDÃO FÍSICA}

No contexto escolar, surgiram correlações significativas e positivas entre:

- $\quad$ o total de jogos realizados e a força abdominal $(r=0,216, p=$ 0,025);

- o total de jogos realizados e a flexibilidade dos membros inferiores (direita $r=0,241, p=0,012$ ) e (esquerda $r=0,240, p=$ 0,013); 
- o número de jogos desportivos com bola e jogos de descoberta com a velocidade de corrida $(r=-0,372, p=0,000)$ e $(r=$ $-0,215, p=0,025)$, respetivamente.

Fora da escola foram também registados as seguintes associações com significância estatística:

- jogos desportivos com bola e resultados no teste do "vaivém" $(r=0,297, p=0,002)$;

- jogos desportivos com bola e velocidade de corrida (tempo registado: $r=-0,376, p=0,000$ ).

\section{DISCUSSÃO}

As crianças com massa corporal e IMC mais elevados praticam menos jogos de Atividade Física, nomeadamente no que concerne aos jogos com bola (desportivos e tradicionais) e de corrida e perseguição. Os dados realçaram a associação entre o tipo e quantidade de jogos realizados em cada um dos contextos analisados e algumas componentes da aptidão física.

A atividade física, principalmente em crianças e adolescentes, é uma das componentes mais difíceis de medir (Anderson et. al., 2005; Kelly et. al., 2006), mas a importância da sua medição e quantificação será porventura tão grande quanto o grau de dificuldade em concretizá-la com a precisão e o rigor pretendido. No caso concreto do presente estudo, atendendo à especificidade da avaliação dos jogos praticados pelas crianças, e não existindo, segundo o nosso melhor conhecimento, estudos na literatura disponível neste âmbito, torna-se difícil estabelecer comparações.

Adicionalmente, a atividade física das crianças e adolescentes é revestida de aspetos muito particulares, que se caracterizam por rápidas mudanças de atividade, episódios curtos, explosivos e intensos que ocorrem de forma esporádica e envolvem uma diversidade de movimentos (Ott et. al., 2000). Esta preferência por curtos e elevados níveis de intensidade, típicos destas idades, também dificulta o estabelecimento de associações entre os jogos realizados e os níveis de composição corporal. No entanto, de uma forma geral, podemos referir que 
estes resultados corroboram os alcançados em vários estudos, que também salientam uma associação inversa entre os níveis de atividade física e índices de massa corporal (McAdams, 2010; Michaliszyn \& Faulkner, 2010; Sola et. al., 2010).

Relativamente à associação do índice de massa corporal com a prestação em habilidades motoras básicas, fundamentais na prática dos jogos considerados, os estudos apontam que um elevado índice de massa corporal limita o desempenho nas habilidades locomotoras, especialmente naquelas em que o corpo está sujeito a deslocamentos de apreciável distância, acelerações horizontais ou deslocamentos verticais (Okely et. al., 2004; Van Den Tillaar \& Ettema, 2004). Já nas habilidades com bola ou controlo de objetos Fogelholm et. al. (2008) e D'Hondt et. al. (2009) registaram uma associação negativa com o índice de massa corporal, ao contrário de Southall et. al. (2004), que não encontraram nenhuma associação significativa entre estas variáveis.

As correlações significativas e positivas observadas entre os jogos e os níveis de aptidão física das crianças, nomeadamente em parâmetros de força, flexibilidade, capacidade aeróbia e velocidade, vão ao encontro dos resultados obtidos em alguns estudos que referem associações positivas dos níveis de atividade física com a força (Wrotniak et. al., 2006; Sola et. al., 2010), velocidade (Loko et. al., 2003; Wrotniak et. al., 2006; Sola et. al., 2010), flexibilidade (Cavill et. al., 2001), e aptidão cardiorrespiratória (Hands et. al., 2009; Sola et. al., 2010).

Algumas investigações referem ainda uma associação positiva entre a atividade física e a qualidade de desempenho das habilidades motoras fundamentais (Houwen et. al., 2008; Hume et. al., 2008; Williams et. al., 2008; D'Hondt et. al., 2009). O lançamento com bola é uma exceção, não se registando, em alguns casos, uma associação significativa entre esta habilidade e a atividade física (Marta, 2006; Jaakkola et. al., 2009). De referir ainda que o facto de o total de referências aos jogos e desportos realizados fora da escola ter atingido um valor próximo do dobro dos praticados em meio escolar, nomeadamente os jogos de saltos, vai no mesmo sentido dos resultados de Serra e Serra (2007). Efetivamente estes autores reportaram uma diminuição acentuada dos jogos de saltos num período temporal de 15 anos, tendo identificado a mesma supremacia destes jogos no contexto não escolar em relação ao recreio, onde, tal como no presente estudo, o valor se mostrou 
baixo.

Os dados vêm ainda confirmar que os jogos de corrida e perseguição são os mais prevalentes nos dois contextos estudados, tal como nos estudos de Serra (1992) e Rebolo (2012).

De referir algumas limitações do estudo: (i) o facto de a amostra ser pouco alargada e, dada a sua natureza transversal, não permitir estabelecer uma relação causal entre as variáveis estudadas; (ii) Não foi contemplado o tempo despendido em cada atividade; (iii) outros fatores de natureza ambiental e sociocultural podem ter influenciado os resultados.

\section{CONCLUSÃO}

Os dados revelaram o quase desaparecimento de jogos de lançamento e de danças e batimentos rítmicos no tempo livre das crianças, registando-se também valores muito baixos nos de dramatização, noutras práticas lúdicas com bola (exceptuando-se os de cariz desportivo) e nos jogos de saltos realizados em meio escolar. As crianças desta amostra com massa corporal e IMC mais elevados praticam menos jogos de atividade física, nomeadamente no que concerne aos jogos com bola (desportivos e tradicionais) e de corrida e perseguição. Verificou-se uma associação entre o tipo e quantidade de jogos realizados em cada um dos contextos analisados e algumas componentes da aptidão física.

Perante estes resultados, pode considerar-se que jogar com os seus pares, no recreio escolar ou fora dele, além dos benefícios que proporciona a outros domínios do desenvolvimento, desempenha um papel fundamental na melhoria da aptidão física.

\section{BIBLIOGRAFIA}

Adamsen, L., Quist, M., Andersen, C., Møller, T., Herrstedt, J., Kronborg, D., ... Rørth, M. (2009). Effect of a multimodal high intensity exercise intervention in cancer patients undergoing chemotherapy: randomised controlled trial. British Medical Journal, 339, b3410. http://doi.org/10.1136/bmj.b3410 Ahmed R, Thomas W, Yee D, Schmitz K. (2006). Randomized controlled trial of weight training and lymphedema in breast cancer survivors. Journal of Clinical 
Oncology. 24(18):2765-72

American College of Sports Medicine. American College of Sports Medicine position stand. Progression models in resistance training for healthy adults. Med Sci Sports Exerc. 2009 Mar; 41 (3):687-708. doi: 10.1249/MSS.0b0 1 3e3 181915670 Blum, D., Omlin, A., Baracos, V.E., Solheim, T.S., Tan, B.H., Stone, P.,.European Palliative Care Research Collaborative (2011). Cancer cachexia: a sys-tematic literature review of items and domains associated withinvoluntary weight loss in cancer. Critical Reviews in Oncol-ogy/Hematology, 80(1): 11 4-44, DOI: 10.1016/i.critrevonc.2010.10.004. PMID: 21216616

Cheema, B.S., Kilbreath, S.L., Fahey, P.P., Delaney, G.P., \& Atlantis, E. (2008) Progressive resistance training $n$ breast cancer: a systematic review of clinical trials. Breast Cancer Res Treat, 109:9-26. DOI: 10.1007/s1 0549-014-3162-9

Campbell, K.L., Pusic, A.L., Zucker, D.S., McNeely, M.L., Binkley, J.M., Cheville, A.L., \& Harwood, K.J.. (2012). A prospective model of care for breast cancer rehabilitation: function. Cancer, 118:2300-2311

Courneya, K.S., Segal, R.J., Mackey, J.R., Gelmon, K., Reid, R.D., Friedenreich, C.M.,

...McKenzie, D.C. (2007). Effects of Aerobic and Resistance Exercise in Breast Cancer Patients Receiving Adjuvant Chemotherapy: A Multicenter Randomized Controlled Trial. Journal of Clinical Oncology, 25(28), 4396-4404. DOI: 10.1200/ico.2006.08.2024

Falk Dahl, C.A., Reinertsen, K.V., Nesvold, I.L., Fosså, S.D. \& Dahl, A.A.. (2010). A study of body image in long-term breast cancer survivors. Cancer, 116 (15):354957. DOI: 10.1002/CNCR.25251

Hagstrom, A.D., Shorter, K.A. \& Marshall, P.W. (2017). Changes in unilateral upper limb muscular strength and EMG activity following a 16 week strength training intervention survivors of breast cancer. Journal of strength and conditioning research. [ahead of print]. DOI:10.1519/JSC.0000000000001890.

Harrington, S., Padua, D., Battaglini, C., Michener, L.A. (2013). Upper extremity strength and range of motion and their relationship to function in breast cancer surviviors. Physiotherapy theory and practice. 29 (7):513-520. DOI: 10.3109/09593985.2012.757683

Herrero, F., San Juan, A., Fleck, S., Foster, C., \& Lucia, A. (2007). Effects of Detraining on the Functional Capacity of Previously Trained Breast Cancer Survivors. International Journal of Sports Medicine, 28(3), 257-264. DOI: 10.1055/s2006-924348

Hidding, J., Beurskens, C., van der Wees, P., van Laarhoven, H., \& Nijhuis-van der Sanden, MW. (2014). Treatment Related Impairments in Arm and Shoulder in Patients with Breast Cancer: A Systematic Review. Plos ONE, 9(5), e96748. 


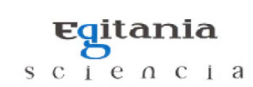

DOI: 10.1371 / journal.pone.0096748

Kwan, M.L., Sternfel, B., Ergas, I.J., Timperi, A.W., Roh, J.M., Hong. C.C.. Kushi, L.H. (2012). Change in physical activity during active treatment in a prospective study of breast cancer survivors. Breast cancer research and treatment. 131 (2): 679-690. DOI: 10.1007/s10549-011-1788-4

M Mayer, E.L. (2013). Early and late long-term effects of adjuvant chemotherapy. American Society of Clinical Oncology educational book. American Society of Clinical Oncology. Meeting, 9-14. DOI: 10.1200/edbook_am.2013.33.9

Reinersten, K.V., Cvancarova, M., Loge, J.H., Edvarsen, H., Wist, E. \& Fossa, S.D. (2010) Predictors and course of chronic fatigue in longterm breast cancer survivirs. Journal of cancer survivorship : research and practice. 4(4):405-41 4. DOI: $10.1007 /$ s $11764-010-0145-7$

Saarto, T., Pentinnen, H., Sievannen, H., Kellokumpu, P., Hakamies Blomquist, L., Nikander, R... Luoma, M.L,. (2012). Effectiveness of a 12 month exercise program on physical performance and quality of life of breast cancer survivors. Anticancer Resume, 32(9), 3875-84

Schwartz, A., Winters-Stone, K., \& Gallucci, B. (2007). Exercise Effects on Bone Mineral Density in Women With Breast Cancer Receiving Adjuvant Chemotherapy. Oncology Nursing Forum, 34(3), 627-633. DOI: 10.1 188/07.onf.627-633 Schmitz KH, Ahmed RL, Hannan PJ, Yee D. (2005). Safety and efficacy of weight training in recent breast cancer survivors to alter body composition, insulin, and insulin-like growth factor axis proteins. Cancer epidemiology, biomarkers and prevention. $14(7): 1672-80$

Schmitz KH, Ahmed RL, Troxel AB, Cheville A, Lewis-Grant L, Smith R, ... Chittams J. (2010). Weight lifting for women at risk for breast cancer-related lymphedema: a randomized trial. Journal of the American Medical Association. 304(24):2699- 705. doi: 10.1001/jama.2010.1837

Schmitz, K.H., Courneya, K.S., Matthews, C., Demark-Wahnefried, W., Galvão, D.A.,, Schwartz, A.L. (2010). American College of Sports Medicine roundtable on exercise guidelines for cancer survivors. Medicine and science in sports and exercise. 42(7): 1 409-26. DOI:10.1249/MSS.0b013e3181e0c1 12

Schmitz KH, Troxel AB, Cheville A, Grant LL, Bryan CJ, Gross CR \& Ahmed RL. (2009). Physical Activity and Lymphedema (the PAL trial): assessing the safety of progressive strength training in breast cancer survivors. Contemporary clinical trials. 30(3):233-45. doi: 10.1016/i.cct.2009.01.001

Siegel, R. L., Miller, K. D. \& Jemal, A. (2015), Cancer statistics, 2015. CA Cancer Journal for Clinicians, 65: 5-29. doi:10.3322/caac.21254

Winters-Stone, K., Laudermilk, M., Woo, K., Brown, J., \& Schmitz, K. (2014). Influence of weight training on skeletal health of breast cancer survivors with or at risk for breast cancer- related lymphedema. Journal of Cancer Survivorship, 
8(2), 260-268. DOI:10.1007/s1 1764- 013-0337-z

WHO. (2010). Global Recommendations on Physical Activity for Health. Ed. World Health Organization; Geneva. pp 18-33. ISBN: 9789241599979 http://

apps.who.int/iris/bitstream/10665/44399/1/9789241599979_eng.pdf 